\title{
Non-modal instabilities of two-dimensional disturbances in plane Couette flow of a power-law fluid
}

\author{
Rong Liu, Qiusheng Liu* \\ Key Laboratory of Microgravity (National Microgravity Laboratory), Institute of Mechanics, Chinese Academy of Sciences, Beijing 100190, China
}

\section{A R T I C L E I N F O}

\section{Article history:}

Received 6 August 2009

Received in revised form 5 June 2010

Accepted 9 June 2010

\section{Keywords:}

Power-law fluid

Instabilities

Non-normal mode

Pseudospectra

\begin{abstract}
A B S T R A C T
Instabilities of fluid flows have traditionally been investigated by normal mode analysis, i.e. by linearizing the equations of flow and testing for unstable eigenvalues of the linearized problem. However, the results of eigenvalue analysis agree poorly in many cases with experiments, especially for shear flows. In this paper we study the instabilities of two-dimensional Couette flow of a polymeric fluid in the framework of non-modal stability theory rather than normal mode analysis. A power-law model is used to describe the polymeric liquid. We focus on the response to external excitations and initial conditions by examining the pseudospectra structures and the transient energy growths. For both Newtonian and non-Newtonian flows, the results show that there can be a rather large transient growth even though the linear operator of Couette flow has no unstable eigenvalue. The effects of non-Newtonian viscosity on the transient behaviors are examined in this study. The results show that the "shear-thinning/shear-thickening" effect increases/decreases the amplitude of responses to external excitations and initial conditions.
\end{abstract}

(C) 2010 Elsevier B.V. All rights reserved.

\section{Introduction}

Polymeric liquids associated with shear flows are commonly found in industrial applications as well as in natural processes. These structurally complex polymeric liquids have behaviors different from that of Newton fluids because of non-Newtonian effects [1]. The instabilities of flow play important roles in many industrial processes, because the growth of disturbances of flow may result in more complicated flow patterns. Non-Newtonian effects on the instability characteristics of polymeric liquids are of particular interest for technology importance.

The equation governing the first order disturbances for a fluid system is in the form of a linear dynamical system:

$$
\frac{d \mathbf{u}}{d t}=-i L \mathbf{u},
$$

in which $\mathbf{u}(t)$ is the state function representing the system at time $t$ and $L$ is the linear operator. In hydrodynamic stability problems, for such a system there are two distinct regions in the evolution of disturbances [2,3]. The first is the asymptotic long time region, and the second is the transient region. In mathematical sense, instability behaviors in the the first region are determined by the eigenvalues of the linear operator $L$. In the second region, the transient growth may be quite substantial, such that the nonlinear region may be reached before the growth of eigenvalue mode. Hence, the

\footnotetext{
* Corresponding author.

E-mail addresses: liurong@imech.ac.cn (R. Liu), liu@imech.ac.cn (Q. Liu).
}

eigenvalues of the system are insufficient to predict to instability behavior of the transient region.

Until now, the eigenvalue analysis is the principal tool in determining the stability characteristics of a fluid system. In this approach the non-linear equations are linearized about the basic solution, and the stability is then determined by examining the eigenvalues of linear operator $L$. If there is an eigenvalue with positive imarginary part, then the corresponding eigenvector exponentially increases with time, and the flow is linearly unstable. The eigenvalue analysis [4] has successfully predicted the instability behaviors for some fluid systems, such as Rayleigh convection and Taylor Couette flow. However, for other instability problems, particularly the instabilities in shear flows, this approach fails to match most experimental results. For example, eigenvalue analysis shows that plane Couette flow and pipe Poiseuille flow are always stable to small disturbances for all Reynolds numbers. However, experimental studies show that Couette flow becomes unstable at Reynolds number $R e=360$ and pipe Poiseuille flow at $R e=2000$. Such discrepancies lead to reexamination of the results of traditional eigenvalue analysis and a novel way, i.e. non-normal stability theory, of describing stability of fluids. The non-normal mode analysis is based on the fact that even if all of the eigenvalues of a linear operator are distinct and lies inside the stable plane, inputs to the system may be amplified by a rather large factor if the operator $L$ is non-normal, i.e. $L L^{*} \neq L^{*} L$. Here ${ }^{*}$ denotes the Hermit transpose. The elements of non-normal stability theory can be referred in the books by Trefethen and Embree [5], and by Schmid and Henningson [6]. We should note that the non-normal analysis is only valid locally and the non-normal growth mechanism does 
not predict global features of transition to turbulence which is essentially a non-linear process. Waleffe $[7,8]$ has made important works on transition in shear flows from the viewpoint of nonlinearity. It is argued that transition is a part of the self-sustaining process by which finite-amplitude solutions are sustained.

Stabilities of non-Newtonian fluids involving shear flows have given rise to great scientific interest. Instabilities of plane Couette flow and Poiseuille flow of non-Newtonian fluid systems have been studied by many authors [9-11]. However, a careful look at previous works on these subjects indicates that most of instability analyses are in the framework of traditional eigenvalue analysis. Recently, some authors have studied the non-modal stability for various non-Newtonian fluid systems [12-14].

Motivated by previous works, we reexamine the instabilities of plane Couette flow of a polymeric liquid using the non-normal stability theory. One of the most important characteristics of macromolecular fluids is the non-Newtonian viscosity, i.e. the fact that the viscosity of the fluid changes with the shear rate. The generalized Newtonian model has been widely used to describe the shear rate dependence of the viscosity. Various forms, such as the Power-law mode and the Carreau model, of the generalized Newtonian model can be referred in the book by Bird et al. [16]. Recently, Chikkadi et al. [14] have studied the effect of viscosity stratification on the transition to turbulence using the Carreau model. Although the generalized Newtonian fluid is of great value in engineering problems, its use is strictly limited to steady-state shearing flows. For unsteady non-Newtonian flows, the elastic response of the fluid becomes important. The Maxwell model containing two constants is widely used to describe the linear viscoelastic fluid [16]. The Oldroyd models can be used to describe the non-linear viscoelastic non-Newtonian fluids [16]. Hoda et al. [17] have investigated frequency responses of streamwise-constant perturbations in channel flows of of Oldroyd-B fluids. For unsteady non-Newtonian flows, both the viscosity and the elasticity will play role in determining the stability of the flow. The influence of viscoelasticity on the transient behaviors of non-Newtonian flows are somewhat complicated. When investigating the problem, it is helpful to look at different aspects of the problem separately whenever possible. In the present problem, we only consider the linear transient behavior of infinitesimal small disturbance upon a steady basic state. It is reasonable to assume that the perturbed state of the flow does not depart far away from the basic state. Thus, we only study the influences of the non-Newtonian viscosity on the transient behaviors and neglect the influence of the elasticity. In the present study, we use the power-law model to describe the polymeric liquid and focus on the effects of non-Newtonian viscosity, i.e. "shear-thinning" and "shear-thickening", on the short time transient rather than eigenvalue mode instabilities.

The paper is organized as follows. In Section 2, the controlling equations of the physical model are established. In Section 3 , we give a brief description of the non-modal stability theory. The numerical methods of computing pseudospectra and transient growth $G(t)$ are presented in Section 4. In Section 5, we present the results and discussions. Finally, in Section 6, we summarize the results and present the conclusions.

\section{Mathematical formulation}

We consider an incompressible power-law fluid bounded by two infinite parallel plates moving in opposite directions with velocities $\pm U$, as illustrated in Fig. 1 . The coordinate system $(x, y, z)$ is chosen such that the origin is a midway between the plates with the $x$ axis in the direction of basic flow and the $z$ axis perpendicular to the plates. The two plates are located at $z= \pm d$. The parallel basic flow $\bar{u}(z)$ in the $x$-direction only depends on $z$.

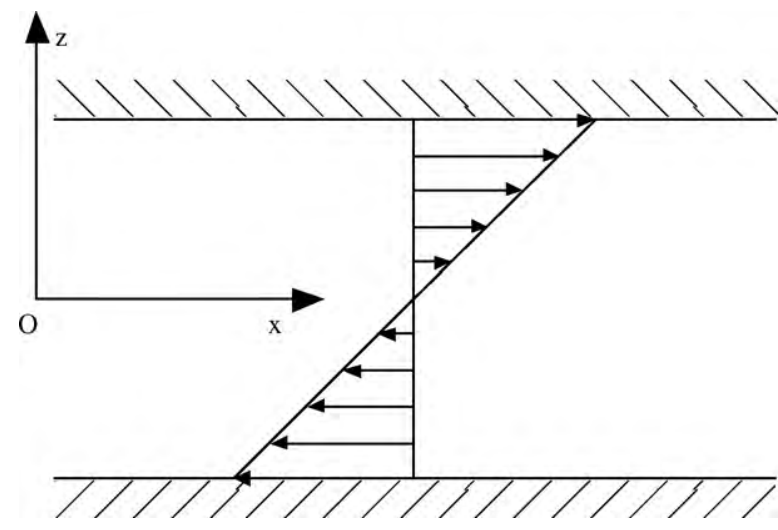

Fig. 1. Geometry and coordinate system for plane Couette flow of a power-law fluid.

For plane shear flow, the linear stability of the streamwise can be described by the Orr-Sommerfeld equation, and the spanwise and three-dimensional disturbances can be described by the Orr-Sommerfeld and Squire equations. For Couette and Poiseuille flows, Squire's theorem [18] implies that the critical Reynolds number for two-dimensional disturbances is lower than the that for three-dimensional ones. So, for normal mode analysis one only need to study the two-dimensional case of streamwise disturbance. We should note that Squire's theorem is only valid for eigenvalue problems. Strictly speaking, in order to seek the optimal transient growth, investigating the non-modal stability of three-dimensional disturbances is indispensable. For Couette and Poiseuille flows of Newtonian fluids, spanwise disturbances have the optimal transient growth [19]. Chikkadi et al. [14] have studied the transient growth of the Poiseuille flow using the Carreau model. The authors stated that the optimal transient growth of spanwise disturbances is only slightly influenced by "shear-thinning" effect. We should note that the viscosity perturbations are neglected in Ref. [14]. Nouar et al. [15] have revisited the same problem. Being different to Chikkadi et al. [14], the authors have considered a nonvanishing viscosity disturbance, and obtained different results to that reported in Ref. [14]. However, the influence of non-Newtonian viscosity on the transient growth of the streamwise disturbances has not been reported in Refs. [14,15].

For the present problem, at a fixed Reynolds number, the transient growth of the streamwise disturbances may be far weaker than that of three-dimensional case. Nevertheless, it does not indicates that investigating the response or the transient growth of streamwise disturbances are not important. From an inputout point of view, input of streamwise disturbance is possible. Before we study the transient behavior of three-dimensional disturbances, it is helpful to know the influence of non-Newtonian viscosity on the non-modal stability of streamwise disturbance. In the present paper, we only consider two-dimensional disturbances, even though we are fully aware that three-dimensional disturbances may have a larger amplification. At least, the results of two-dimensional streamwise disturbances predict the lowest bound of the optimal growth of the disturbances propagating in different directions.

\subsection{Controlling equations}

The equations governing the evolution of fluid flow are the Navier-Stokes equations. For an incompressible non-Newtonian fluid, the equations are expressed as:

$$
\begin{aligned}
& \nabla \cdot \mathbf{u}=0, \\
& \frac{\partial \mathbf{u}}{\partial t}+\mathbf{u} \cdot \nabla \mathbf{u}=-\frac{1}{\rho} \nabla p+\nabla \cdot \boldsymbol{\tau},
\end{aligned}
$$


with the boundary conditions

$u(d)=U, \quad v(d)=0, \quad w(d)=0$,

$u(-d)=-U, \quad v(-d)=0, \quad w(-d)=0$,

where $t$ is the time, $\mathbf{u}$ is the velocity vector, $u, v, w$ are the velocity components in the $x, y, z$ directions respectivley, $p$ is the pressure, $t$ is the stress tensor which can be written in a generalized Newtonian form [1]

$\boldsymbol{\tau}=\eta \dot{\boldsymbol{\gamma}}, \quad$ with $\quad \eta=\eta(\dot{\gamma})$

here $\dot{\gamma}=\nabla \mathbf{u}+(\nabla \mathbf{u})^{T}$ is the rate of strain tensor, and the effective viscosity $\eta$ is the function of $\dot{\gamma}$. The magnitude of the rate of strain tensor is defined as:

$\dot{\gamma}=\sqrt{\frac{1}{2}(\dot{\gamma}: \dot{\gamma})}$

There are many expressions to describe the non-Newtonian viscosity function $\eta(\dot{\gamma})$. For polimeric liquid, we choose the twoparameter power-law expression for $\eta(\dot{\gamma})$

$\eta=m \dot{\gamma}^{n-1}$,

in which $m$ and $n$ are constants characterizing the fluid. In processing range of many polymeric liquids the power-law is a good approximation. A "shear-thinning" fluid has $n<1$, and a "shearthickening" fluid has $n>1 . n=1$ reduces to the Newtonian case. Nearly all non-Newtonian fluids show "shear-thinning", only some particularly concentrated suspensions show regions of "shearthickening" [20].

The evolution equations for the disturbances can be derived by considering a basic state $(\overline{\mathbf{u}}, \bar{p})$ and the perturbed state $(\mathbf{u}, p)=$ $\left(\overline{\mathbf{u}}+\mathbf{u}^{\prime}, \bar{p}+p^{\prime}\right)$. Here the prime denotes disturbed variable. For both states, the Navier-Stokes equations are satisfied.

\subsection{Basic state}

In the basic state, the velocity components and the magnitude of $\dot{\gamma}$ are:

$\mathbf{u}=\left(\frac{U z}{d}, 0,0\right), \quad \overline{\dot{\gamma}}=\frac{U}{d}$

The rate of strain tensor and the stress tensor are:

$\overline{\dot{\gamma}}=\left(\begin{array}{ccc}0 & 0 & \frac{U}{d} \\ 0 & 0 & 0 \\ \frac{U}{d} & 0 & 0\end{array}\right), \quad \overline{\boldsymbol{\tau}}=\left(\begin{array}{ccc}0 & 0 & m\left(\frac{U}{d}\right)^{n} \\ 0 & 0 & 0 \\ m\left(\frac{U}{d}\right)^{n} & 0 & 0\end{array}\right)$.

\subsection{Linearized equations}

Subtracting the equations for the basic and perturbed state, the linearized evolution equations for disturbances can be written as:

$\nabla \cdot \mathbf{u}^{\prime}=0$

$\frac{\partial \mathbf{u}^{\prime}}{\partial t}+\overline{\mathbf{u}} \cdot \nabla \mathbf{u}^{\prime}+\mathbf{u}^{\prime} \cdot \nabla \overline{\mathbf{u}}=-\frac{1}{\rho} \nabla p^{\prime}+\nabla \cdot\left(\bar{\eta} \dot{\gamma}^{\prime}\right)+\nabla \cdot\left(\eta^{\prime} \bar{\gamma}\right)$,

in which

$\nabla \cdot\left(\bar{\eta} \dot{\gamma}^{\prime}\right)=m \overline{\dot{\gamma}}^{n-1} \nabla^{2} \boldsymbol{u}^{\prime}$,

$\nabla \cdot\left(\eta^{\prime} \dot{\gamma}\right)=m(n-1) \overline{\dot{\gamma}}^{n-1}\left[\frac{\partial \gamma_{x z}^{\prime}}{\partial z} \mathbf{e}_{x}+\frac{\partial \gamma_{x z}^{\prime}}{\partial x} \mathbf{e}_{z}\right]$,

here $\gamma_{x z}^{\prime}=\partial w^{\prime}\left|\partial x+\partial u^{\prime}\right| \partial z, \mathbf{e}_{x}$ and $\mathbf{e}_{z}$ denote the unit vectors point in the $x$ and $z$ directions.
For Couette flow, the non-Newtonian fluid has the same basic velocity with Newtonian ones. The last term in Eq. (12) denotes the departure from Newtonian fluid behavior. In Eq. (14), we note that this additional term only appears in the $x$ and $z$ momentum equations. Thus, the non-Newtonian effect of nonconstant viscosity is anisotropic.

The two-dimensional streamwise disturbances are expanded in Fourier form:

$f^{\prime}(x, z, t)=f(z, t) \exp (i \alpha x)$,

here $\alpha$ is the streamwise wavenumber.

The controlling equations are nondimensionalized with length scale $d$, velocity scale $U$, time scale $d / U$. Using the continuity equation and eliminating the pressure, we obtains the Orr-Sommerfeld equation for plane Couette flow:

$\frac{\partial}{\partial t}\left(D^{2}-a^{2}\right) w+i a z\left(D^{2}-a^{2}\right) w=\frac{1}{R e}\left[n D^{4}+2(n-2) a^{2} D^{2}+n a^{4}\right] w$,

with boundary conditions

$w( \pm 1)=D w( \pm 1)=0$,

in which $D$ denotes $\partial / \partial z$, the Reynolds number is defined as:

$R e=\frac{\rho U d}{m}\left(\frac{d}{U}\right)^{n-1}$

\section{Non-modal stability theory}

In non-modal stability theory, stability is redefined in a broader sense as the response to general input variables, including initial conditions, impulsive and continuous external excitations. In mathematic sense, an impulsive excitation is equivalent to an initial condition. In this section, we will give a brief description of the nonmodal theory. More details can be referred in the books by Trefthen et al. [5] and by Schmid et al. [6]. As most of previous works on the subject of non-modal analysis on hydrodynamic stabilities, we are interested in responses to continuous external excitations and to initial conditions.

Now we begin with the responses to external excitations. Suppose a fluid system is driven by a signal of the form:

$\mathbf{V}(x, y, z, t)=\exp (-i \omega t) \mathbf{v}(x, y, z)$,

in which $\omega$ is the complex frequency. Then the response $U(x, y, z, t)$ and the input signal $V$ satisfy the equation:

$\frac{d U}{d t}=-i L U+\exp (-i \omega t) \boldsymbol{v}$.

From Eq. (20), we obtain the response $U(x, y, z, t)$ in the form of

$U(x, y, z, t)=i \exp (-i \omega t) \mathbf{u}=i \exp (-i \omega t)(\omega I-L)^{-1} \mathbf{v}$,

here $I$ is the identical matrix. The solution operator $(\omega I-L)^{-1}$ is known as the resolvent. We denote the maximum amplification of a disturbance at frequency $\omega$ by $R(\omega, \alpha) \cdot R(\omega, \alpha)$ is equal to the norm of the resolvent and expressed as:

$R(\omega, \alpha)=\sup _{\mathbf{v} \neq 0} \frac{\|\mathbf{u}\|}{\|\mathbf{v}\|}=\left\|(\omega I-L)^{-1}\right\|$,

here $\|\cdot\|$ denotes a norm on $L$, "sup" denotes maximum. An eigenvalue of $L$ is a number $\omega$ such that $\left\|(\omega I-L)^{-1}\right\|=\infty$. Generalizing this result leads naturally to the definition of " $\varepsilon$-pseudospectra" [5].

For each $\varepsilon>0$, the $\varepsilon$-pseudospectra $\sigma_{\varepsilon}(L)$ of an operator $L$ is the set of $\omega \in \mathbb{C}$ such that

$\left\|(\omega I-L)^{-1}\right\| \geq \varepsilon^{-1}$. 
Pseudospectra can also be defined in other equivalent ways [5]. For a normal operator $L$, the 2-norm of the resolvent $R(\omega, \alpha)=$ $\left\|(\omega I-L)^{N-1}\right\|_{2}$ is given by $R(\omega, \alpha)=\sup _{\sigma \in \Lambda(L)}|\omega-\sigma|^{-1}$, where $\Lambda(L)$ is the spectrum of $L$ and $|\cdot|$ denotes the distance. When $\omega$ is far from the spectrum the resolvent norm of a normal operator is small. However, for a non-normal operator $L$, the norm of the resolvent can be large even when $\omega$ is far from the spectrum.

Quest for maximum amplification of initial condition is of particular interest in many hydrodynamic stability problems. For a linearized fluid system:

$$
\frac{d \mathbf{u}}{d t}=i L \mathbf{u}
$$

if the operator $L$ has no time-dependence, the solution has the form:

$\mathbf{u}(t)=e^{-i L t} \mathbf{u}(0)$.

The maximum amplification of initial condition has the form:

$$
G(t)=\sup _{\mathbf{u}(0) \neq 0} \frac{\|\mathbf{u}(t)\|^{2}}{\|\mathbf{u}(0)\|^{2}}=\left\|e^{-i L t}\right\|^{2} .
$$

\subsection{Choice of measure}

We note that both the pseudospectral and the growth function are dependent on the definition of norm. From mathematical and physical consideration, an appropriate measure of the disturbance is indispensable. Mathematically speaking, it is convenient to take $\|\cdot\|$ to be 2 -norm and the corresponding inner product is

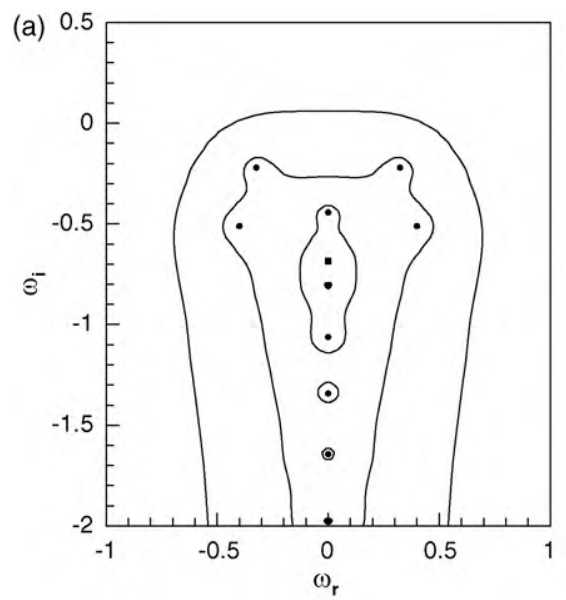

defined as:

$(\mathbf{u}, \mathbf{v})=\mathbf{v}^{*} \mathbf{u}$

here ${ }^{*}$ denotes the conjugate transpose. The energy norm can be introduced as:

$\|\left.\mathbf{v}\right|^{2}=\int_{-1}^{1}\left(|u|^{2}+|v|^{2}+|w|^{2}\right) d z$,

here $\mathbf{v}=(u, v, w)$ are the amplitude of velocity. After introducing a Fourier mode associated with the streamwise wavenumber $\alpha$, for two-dimensional disturbances the energy norm is in the form of

$\|\mathbf{v}\|^{2}=\frac{1}{\alpha^{2}} \int_{-1}^{1}\left(|D w|^{2}+\alpha^{2}|w|^{2}\right) d z$.

As seen in Section 4, the energy norm can be handled within the framework of the 2-norm by introducing an energy matrix.

\section{Numerical method}

We implement a Chebyshev collocation method to solve the pseudo-eigenvalue problem. The variable $w$ is expanded as:

$w=\sum_{n=0}^{N} a_{n} T_{n}(z)$,

(b)

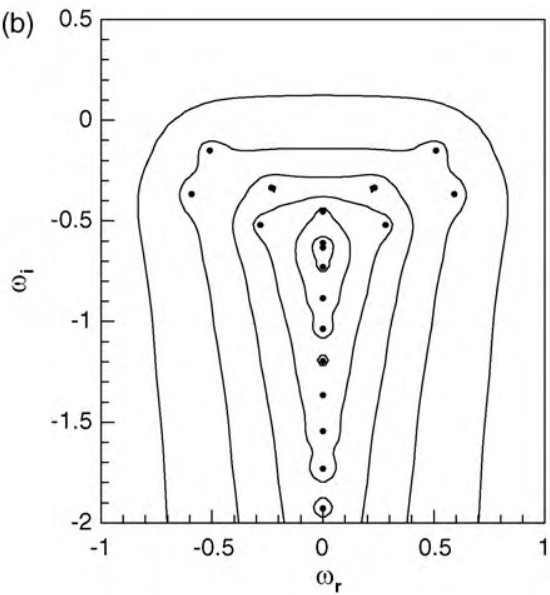

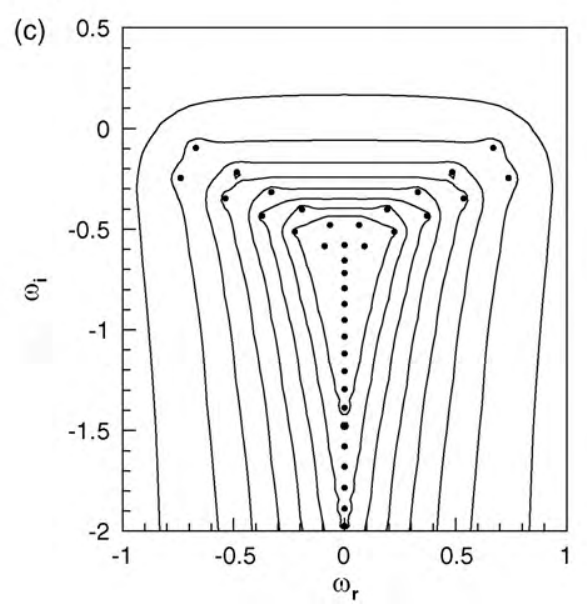

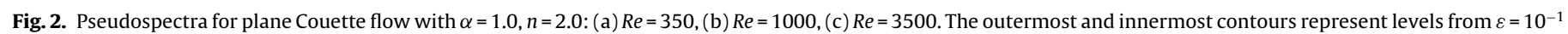
to $10^{-3}$ in (a), $10^{-6}$ in (b) and $10^{-8}$ in (c). 
in which $T_{n}$ denotes the $n$th Chebyshev polynomial. Using the Chebyshev series (30), Eq. (16) can be written in vector form:

$\frac{d}{d t} \mathbf{w}=L_{o s} \mathbf{w}$,

in which $\mathbf{w}=\left(a_{0}, \ldots, a_{N}\right)$. This system of equations is required to solve for $N+1$ unknowns. Chebyshev-Tau method and collocation method have been used to solve eigenvalue problems by many authors. The numerical method for the general eigenvalue problem in the form of $A x=\lambda B x$ are described in the book by Canuto et al. [22]. In our problem, after we implement the boundary conditions, the matrix $B$ should remain to be non-singular. The detail of implementation of the boundary conditions can be refer in Ref. [6]. In our computations, $N=80$ is enough to obtain satisfactory results.

We substitute Eq. (30) into Eq. (29), the energy norm of $v$ is expressed as:

$\|\mathbf{v}\|^{2}=\mathbf{w}^{*} \mathbf{E w}$

The matrix $\mathbf{E}$ is symmetric and can be decomposed in the form $\mathbf{E}=\mathbf{M}^{*} \mathbf{M}$. The energy norm is expressed as:

$\|\mathbf{v}\|^{2}=(\mathbf{M w}, \mathbf{M w})$.

In our computation, let $\mathbf{x}=\mathbf{M w}$. Thus, the energy norm of $\mathbf{v}$ is equivalent to the 2-norm of $\mathbf{x}$, and the $\mathrm{O}-\mathrm{S}$ equation is in the form of

$\frac{d}{d t} \mathbf{x}=-i \mathbf{M} L_{o s} \mathbf{M}^{-1} \mathbf{x}$

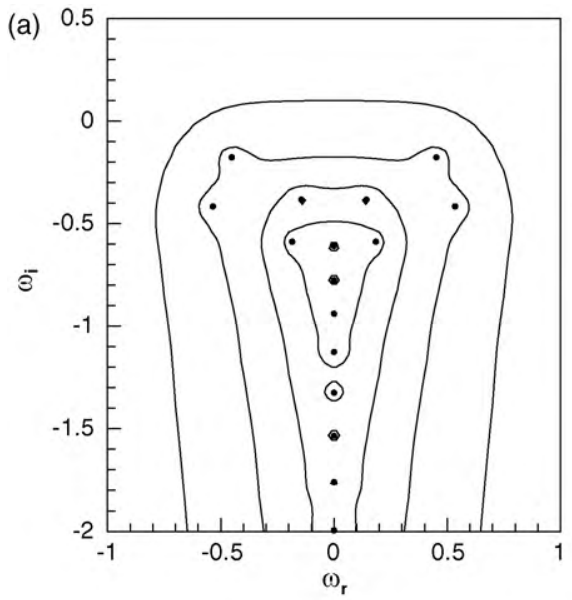

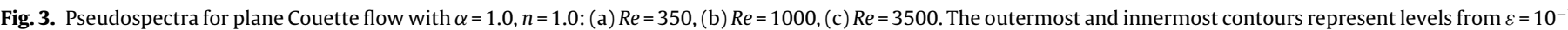
to $10^{-4}$ in (a) and $10^{-8}$ in (b) and (c).
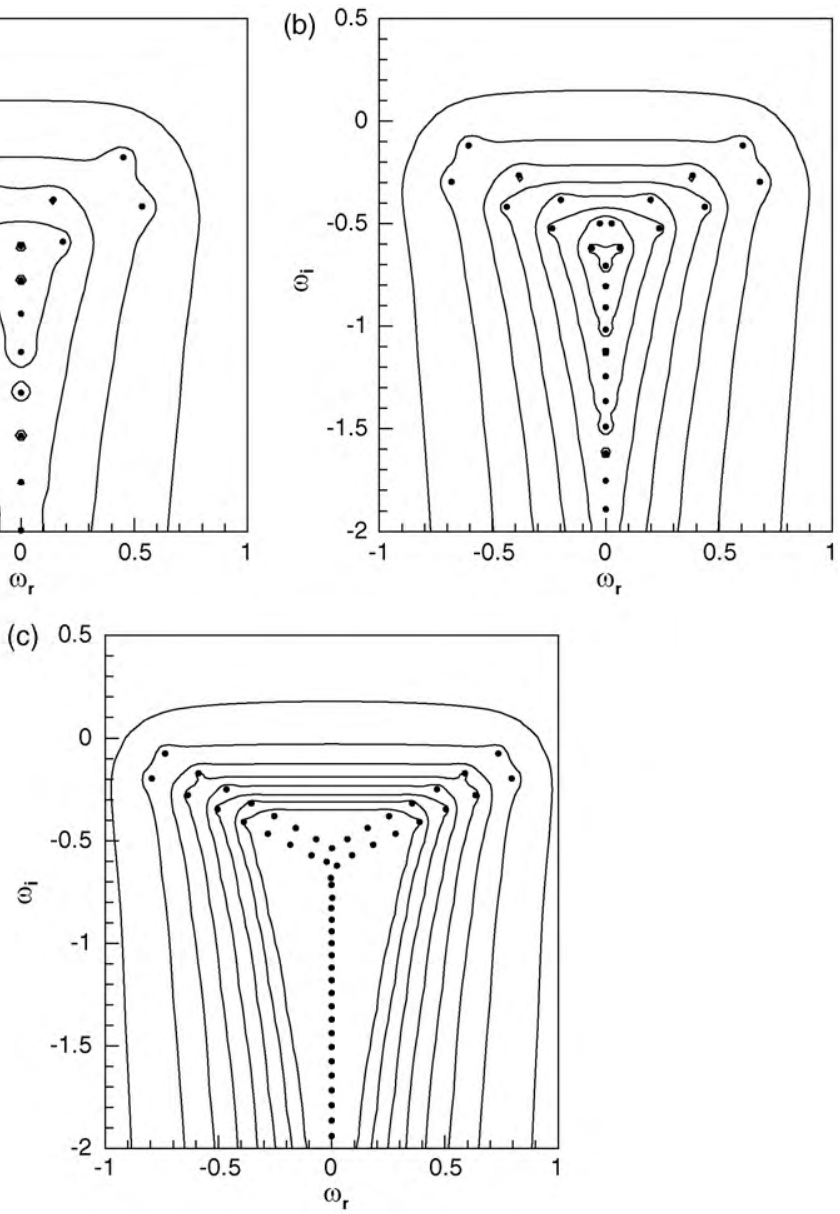

We compute the norm of the solvent by using the relation:

$\left\|(\omega I-L)^{-1}\right\|_{2}=\left[s_{\min }(\omega I-L)\right]^{-1}$,

where $L=\mathbf{M} L_{o s} \mathbf{M}^{-1}, s_{\min }(\omega I-L)$ denotes the smallest singular value of $\omega I-L$. Thus, the pseudospectra can be obtained by solving the singular value problem. It is convenient to compute the entire set of singular values of $\omega I-L$ using the SVD function in matlab or library software such as LAPACK.

The growth function $G(t)$ associating with the energy norm can be computed using the method in Ref. [2]. In this approach we only need the eigenvalues and corresponding eigenvectors. The function $G(t)$ is approximated by

$G_{K}=\left\|F e^{-i \Lambda_{K} t} F^{-1}\right\|_{2}^{2}$,

where $\Lambda_{K}$ is the matrix of dimension $K$ with the first $K$ eigenvalues on the diagonal. $F$ can be obtained by $A=F^{*} F$ in which the components of $A$ are the inner product of the eigenvector:

$A_{j l}=\left(q_{j}, q_{l}\right)$.

The growth function can also be computed directly by

$G(t)=\left\|e^{-i L t}\right\|_{2}^{2}$.

Our computations indicate that these two method yield almost indistinguishable results except for $t \rightarrow 0$.

\section{Results and discussions}

The eigenvalues only predict the long time instability behaviors. Only for normal operators, eigenvalues is sufficient to describe the 

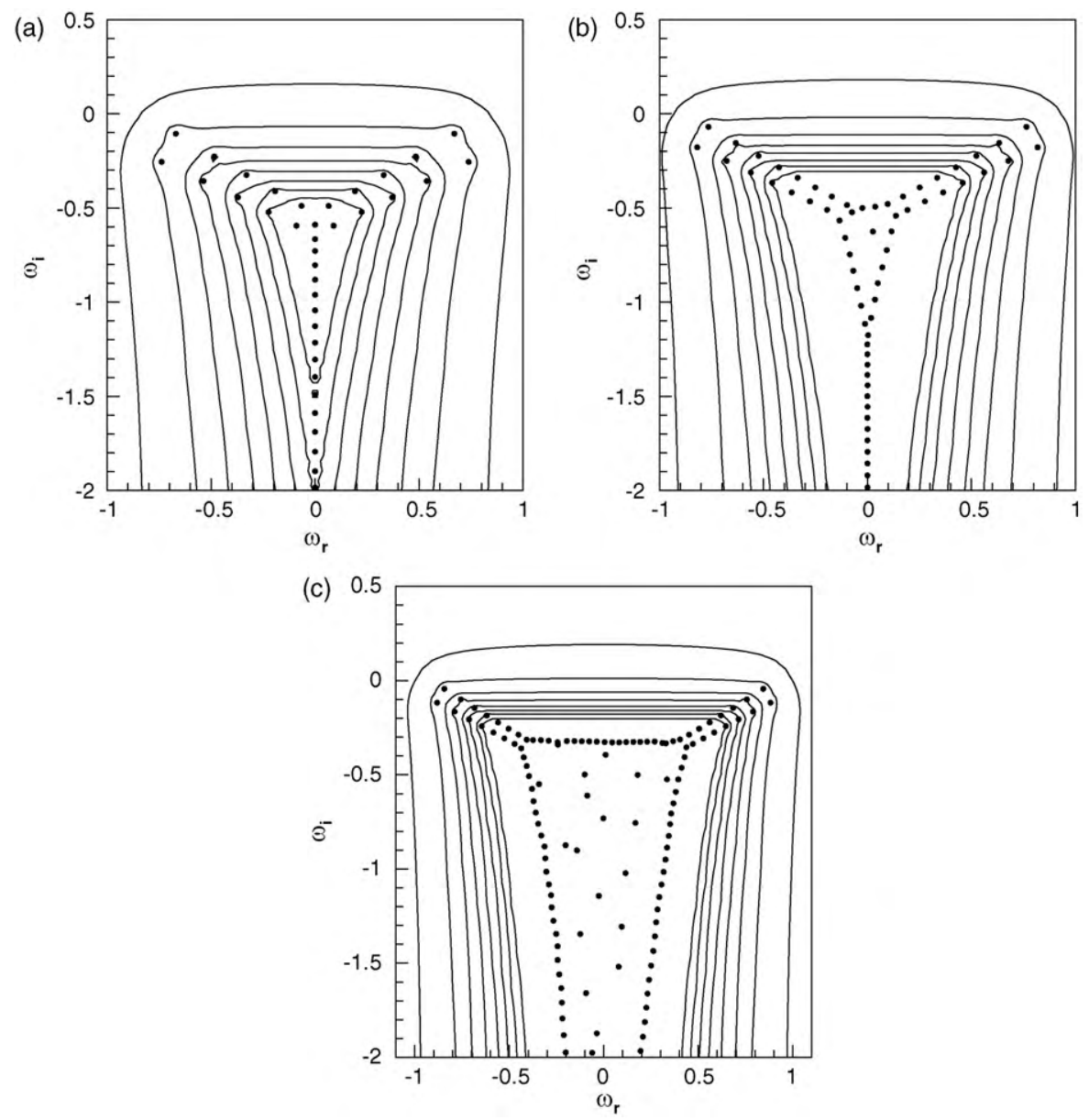

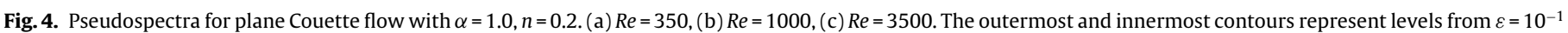
to $10^{-8}$.

instability behaviors for all time. For the present problem, the operator is of non-normal type. We will study the transient behaviors of the responses to external excitations and initial conditions. In mathematical meaning, the resolvent norm and the growth function represent the amplification of response to external forcing and initial condition, respectively.

\subsection{Spectra and pseudospectra}

The instability behavior depends not only on the eigenvalues, but on the regions where the resolvent norm is large. For the present problem, examining the structure of pseudospectra is helpful to understand the influences of the Reynolds number and the effect of non-Newtonian viscosity on the transient behavior.

Figs. 2-4 plot pseudospectral boundaries together with the spectra in the $\omega$ plane for $n=2.0,1.0,0.2$ and $\alpha=1.0$ with Reynolds numbers $R e$ in the range of 350-3500. The real part of the complex frequency is denoted by $\omega_{r}$ and the imaginary part by $\omega_{i}$. In these figures, $n=2.0,1.0,0.2$ correspond "shear-thickening", Newtonian and "shear-thinning" cases, respectively. For Newtonian fluid, these Reynolds numbers roughly span the range from occasional turbulence to unavoidable turbulence. As expected, the system does not permit unstable spectra for both Newtonian and non-Newtonian fluids.

In Fig. 2 for the "shear-thickening" case of $n=2.0$, the spectra consist of three main branches, i.e. the left, middle, and right branches. The left and the right branches are symmetric about $\omega_{r}=0$. Comparing Fig. 2(a)-(c), it is observed that the number of eigenvalues on each branch depends on the Reynolds number. In
Fig. 2(a) for $R e=350$, there are only two eigenvalues in the left or the right branch. As Re increases to 3500 , the number of eigenvalues in the left branch or the right branches increases to 10. In Fig. 2(a)-(c), the inner boundaries of pseudospectra are located near the intersection of the three branches. In Fig. 2(a) the inner boundary is $\varepsilon=10^{-3}$, and the lines of $\varepsilon \leq 10^{-4}$ shrink to the points of eigenvalues. In Fig. 2(c), the inner boundary of $\varepsilon=10^{-8}$ covers an appreciable region near the intersection of the three branches. The pseudospectra in Fig. 2(a)-(c) show that with the increase of Reynolds number, the intersection region of the three branches becomes more sensible to perturbations.

In Fig. 3, the structures of spectra for $n=1.0$ are qualitatively similar to those for $n=2.0$ in Fig. 2 . Comparing the spectra and the boundary of pseudospectra in Fig. 3(a)-(c) to that in Fig. 2(a)-(c) respectively, we observe that each branch of $n=1.0$ has a larger number of spectra, and the vicinity of intersection of the branches of $n=1.0$ is more sensible to perturbations than that of $n=2.0$.

As shown in Fig. 4(a) for $n=0.2$, the structure of spectra of $R e=350$ is qualitatively similar to those in Figs. 2 and 3. However, even for such a low Reynolds number, the eigenvalues in the vicinity of the intersection of branches are rather sensible to perturbations. With the increase of the Reynolds number, the sensibility of the spectra in the intersection region dramatically increases. As a result, the symmetry of middle branch is broken and the middle branch becomes a region consist of random spectra.

As the above discussions, the results show that the sensibility of the vicinity of the intersection is dependent on the Reynolds number and the coefficient $n$. The increase of Reynolds number and the "shear-thinning" effect play an active role in increasing 

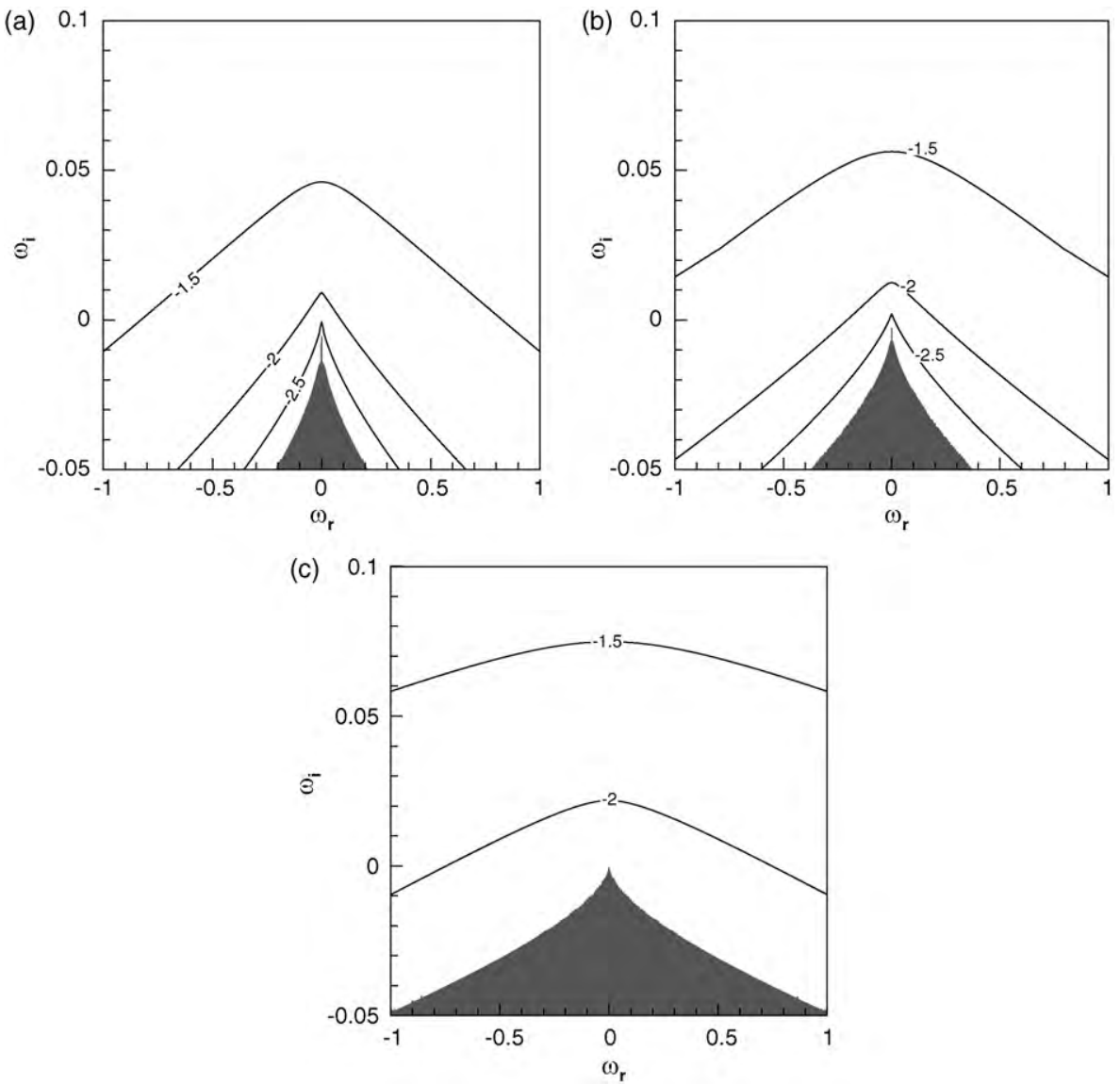

Fig. 5. Pseudospectra of $L$ for two-dimensional plane Couette flow with $R e=3500$. (a) $n=2.0$, (b) $n=1.0$, (c) $n=0.2$. The contours represent the level of log $\varepsilon$.

the sensibility to perturbations and the amplification to external excitations.

\subsection{Resonance of external excitation}

Spectra and pseudospectra provide us with the transient behavior for a given wavenumber $\alpha$. In order to obtain the maximum of resonance to external excitations, we need to examine the global behavior of the operator $L$ for all wavernumbers.

Plots of the pseudospectra of the continuous operator are presented in Fig. 5 for $n=2.0,1.0,0.2$. In Fig. 5, the spectra of two-

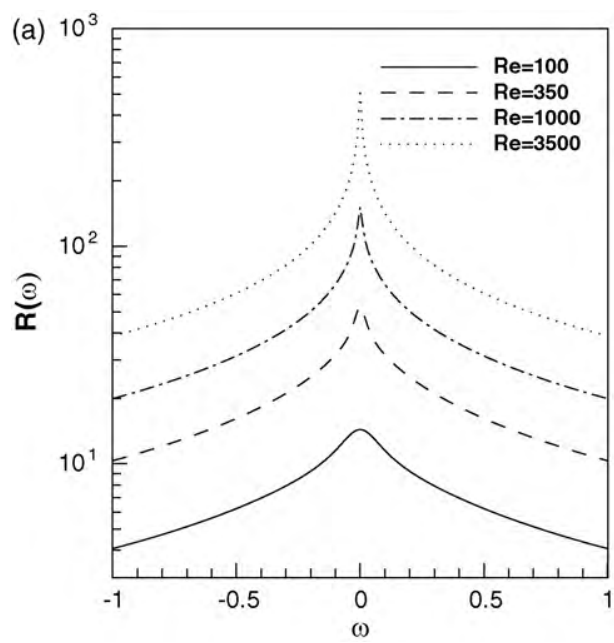

dimensional operator $L$ are confined in the shade region. Trefethen et al. [21] presented the pseudospectra of the three-dimensional operator for Newtonian Couette flow. For each $(\alpha, \beta)$ the the spectrum is discrete. Here $\beta$ is the spanwise wavenumber. The spectra of three-dimensional operator $L$ are continuous. According to Square theorem [18], the two-dimensional instability is more unstable than the three-dimensional counterpart. So, spectra of the three-dimensional operator have the same boundary as that of the two-dimensional one. Here we should note that the spectra of the two-dimensional operator are discrete and only confined in the shade region. The contour of $\varepsilon$ can be interpreted as the

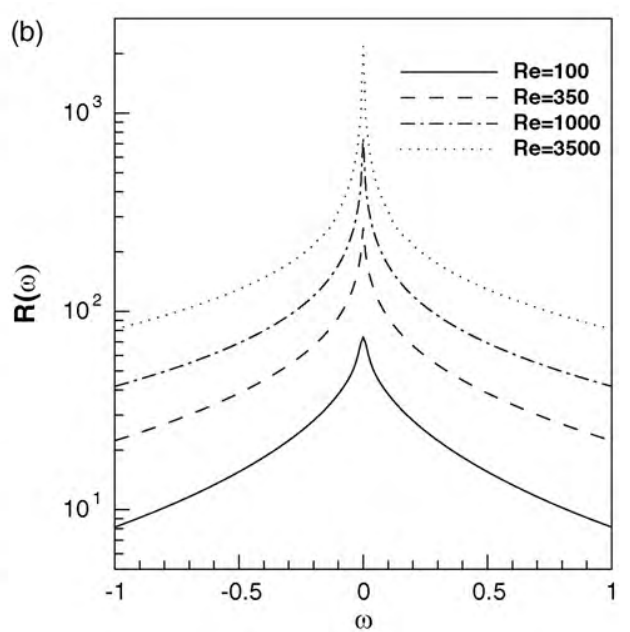

Fig. 6. Pseudoresonance to real frequency for two-dimensional plane Couette flow at various Reynolds numbers. (a) $n=1.0$, (b) $n=0.2$. 


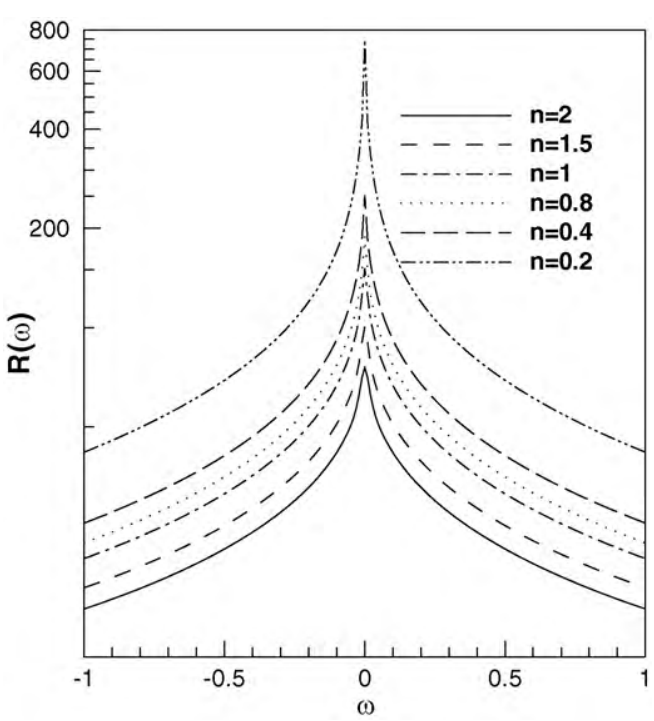

Fig. 7. Pseudoresonance to real frequency for two-dimensional plane Couette flow with various $n$ at $R e=1000$.

points of equal magnitude of resonance to external excitations. It is shown that with the decrease of $n$, the spectral region significantly extends in the stable plane $\left(\omega_{i}<0\right)$. However, unstable eigenvalues are not permitted even when $n$ decreases to 0.2. Apparently, with the decrease of $n$, the pseudospectra of $\varepsilon=10^{-2}, 10^{-1.5}$ protrude more deeply into the unstable plane $\left(\omega_{i}>0\right)$. As shown in Fig. 5(a)-(c), for each $\omega$ the magnitude of resonance $\varepsilon^{-1}$ increases with the decrease of $n$. This indicates that "shear-thinning" effect will amplify the resonances to external excitations.

In the complex $\omega$ plane, we are interested in the resonance of the real axis because it corresponds to external excitations at real frequencies. The maximal amplification of disturbance with a fixed wavenumber has been defined by Eq. (22). We define the maximal resonance of excitations with real frequencies as:

$R(\omega)=\max _{\forall \alpha \in \mathbb{R}} R(\omega, \alpha), \quad \omega \in \mathbb{R}$.

In Fig. 6(a) and (b), the curves of the maximal resonance versus the frequency of external excitation are presented for Newtonian fluid and "shear-thinning" fluid at various Reynolds number from 100 to 3500 . As shown in these figures, the resonances significantly increase with the Reynolds number for both cases of $n=1.0$ and 0.2. It is also observed that the resonance decreases monotonously with the magnitude of frequency. This result indicates that the largest

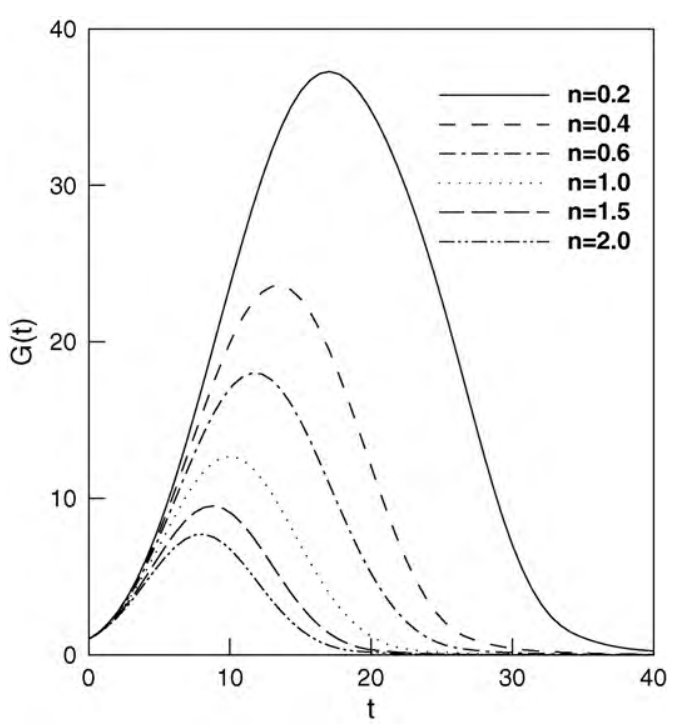

Fig. 9. Transient growth $G$ versus time for two-dimensional plane Couette flow with various $n$ at $R e=1000$.

amplification is achieved for time-independent excitation and the response is more sensible to lower frequency disturbances.

In Fig. 7, the curves of the maximal resonance versus the frequency are presented for different $n$ at the Reynolds number $R e=1000$. As shown in this figure, the maximal resonance occurs at zero frequency for each $n$. The resonance significantly increases with the decrease of $n$ at each frequency. The curves in Fig. 7 show that the "shear-thinning" effect and the "shear-thickening" effect have adverse influences on the resonances to external excitations.

\subsection{Response to initial condition}

In this subsection, we will examine the effects of non-Newtonian viscosity and the Reynolds number on the growth function $G(t)$ of streamwise disturbances. We define the optimal growth $G_{\max }=$ $\max _{t} G(t)$, and denote the optimal time corresponding to $G_{\max }$ by $t_{\text {max }}$. Reddy and Henningson [19] consider three types of behaviors for the Poiseuille flow of Newtonian fluids. If the Reynolds number is less than $\operatorname{Re}_{1}$, then $G(t)<1$ for $t<0$. In this case, $G_{\max }=1$ and $t_{\text {max }}=0$. If the Reynolds number exceeds $R e_{2}$ such that the operator $L$ has an unstable eigenvalue, then $G_{\max }=\infty$ and $t_{\max }=\infty$. If $R e_{1}<R e<R e_{2}$, the flow is linearly stable but has a transient growth.
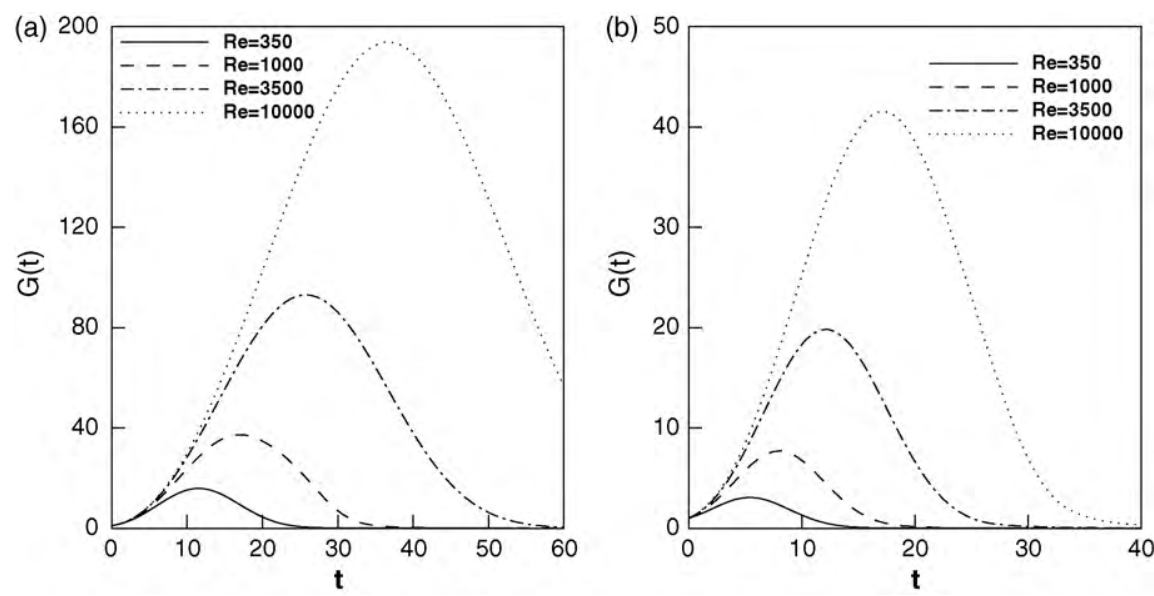

Fig. 8. Transient growth $G$ versus time for two-dimensional plane Couette flow at various Reynolds numbers. (a) $n=0.2$, (b) $n=2.0$. 
For general instability problems, these three types of behavior should be considered. However, for Couette flow the operator has no unstable eigenvalue, so $\operatorname{Re}_{2}=\infty$. For Newtonian Couette flow, we found that $\operatorname{Re}_{1}=57.5$ at $\alpha=1.0$. For non-Newtonian cases, we compute $R e_{1}$ for various $n$ in the range of $0.2 \leq n \leq 2.0$ at $\alpha=1.0$. It is found that $R e_{1}$ and $n$ has approximately a linear relation expressed as:

$R e_{1}=22.6+43.9(n-0.2)$.

In general, $R e_{1}$ is the function of $\alpha$ at a fixed $n$. In the latter part of the present paper, we will present the curves of $R e_{1}$ versus $\alpha$.
Fig. 8(a) and (b) presents the curves of the energy growth function $G(t)$ versus time for "shear-thinning" and "shear-thickening" cases at several typical Reynolds numbers. As shown in these figures, each curve shows that the Couette flow experiences a transient growth and then decays for $t>t_{\max }$. For both cases of $n=2.0$ and $0.2, G_{\max }$ and $t_{\max }$ apparently increase with the growth of the Reynolds number. Fig. 9 presents the curves of $G(t)$ versus time for various $n$ at $R e=1000$. With the decrease of $n$, both $G_{\max }$ and $t_{\max }$ significantly increase.

For two-dimensional flow, it is convenient to introduce the streamfunction to describe the flow structure. In the present prob-
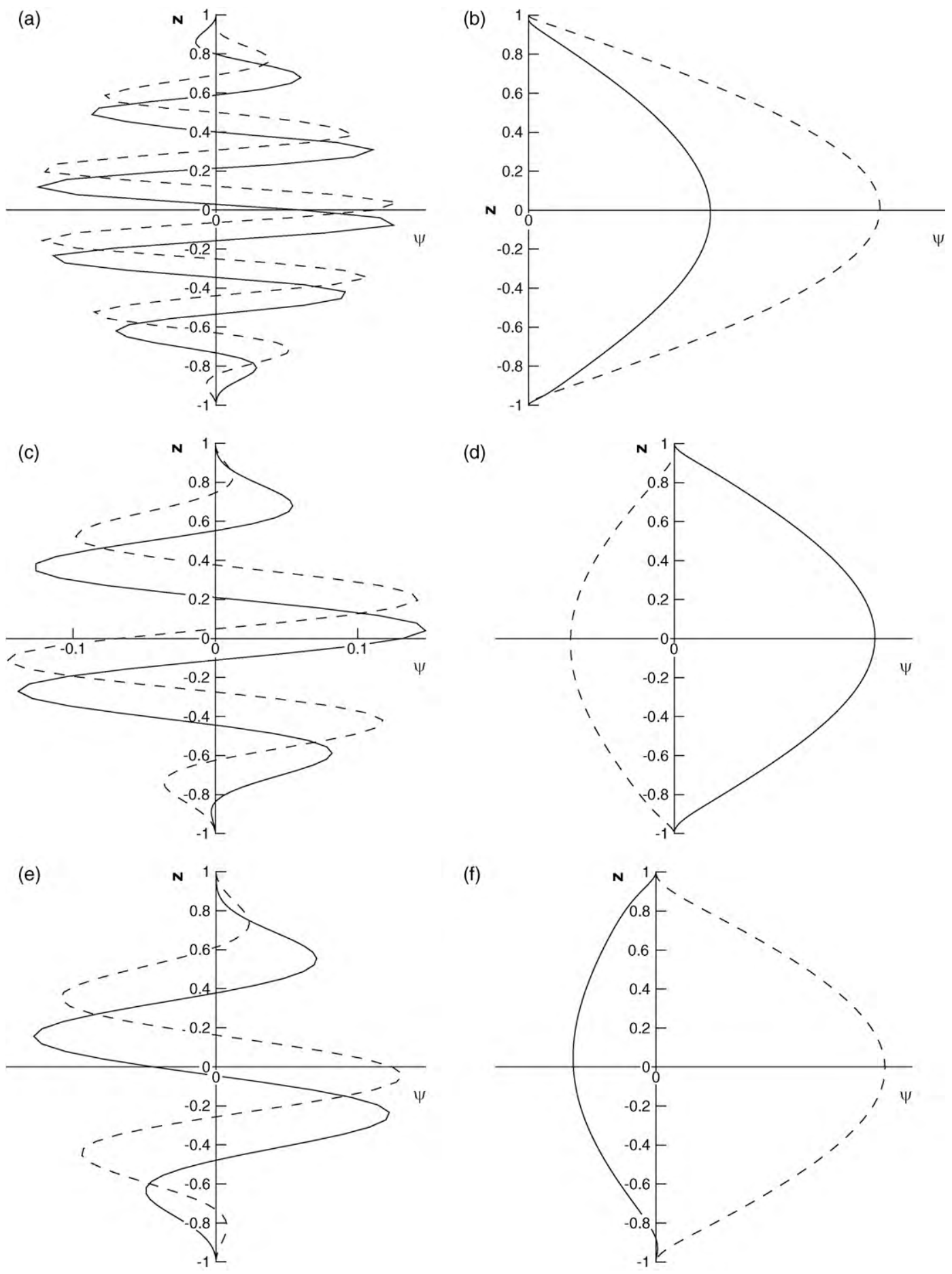

(f)

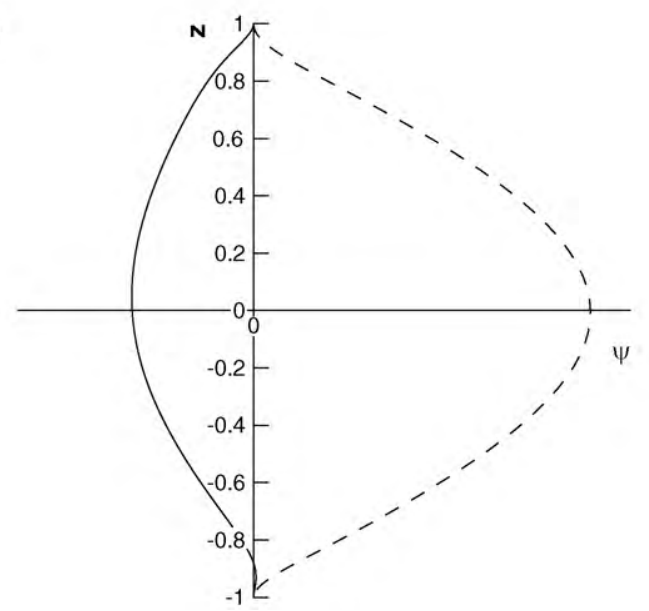

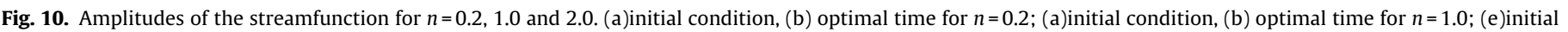
condition, (f) optimal time for $n=2.0$; The other parameters are $\operatorname{Re}=1000$, the streamwise wavenumber $\alpha=1.0$. 
(a)

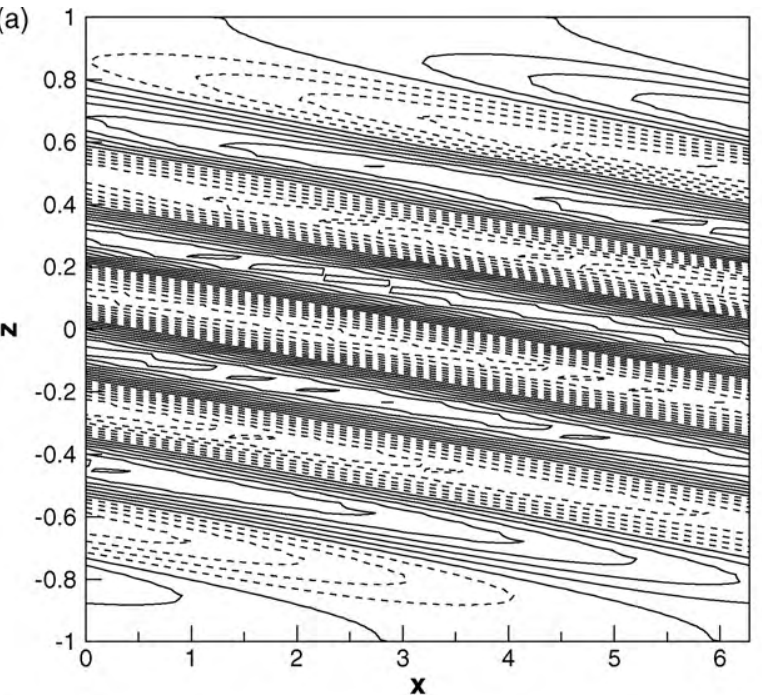

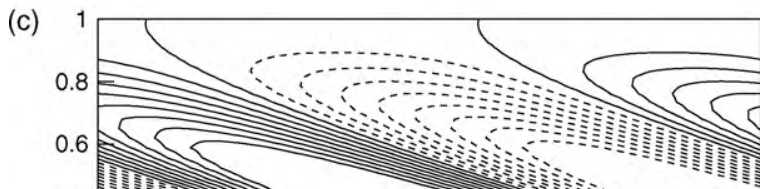
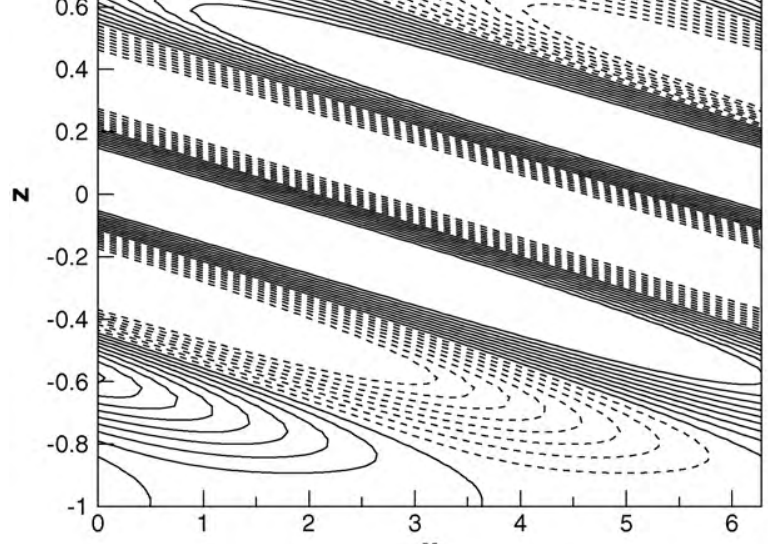

(e)

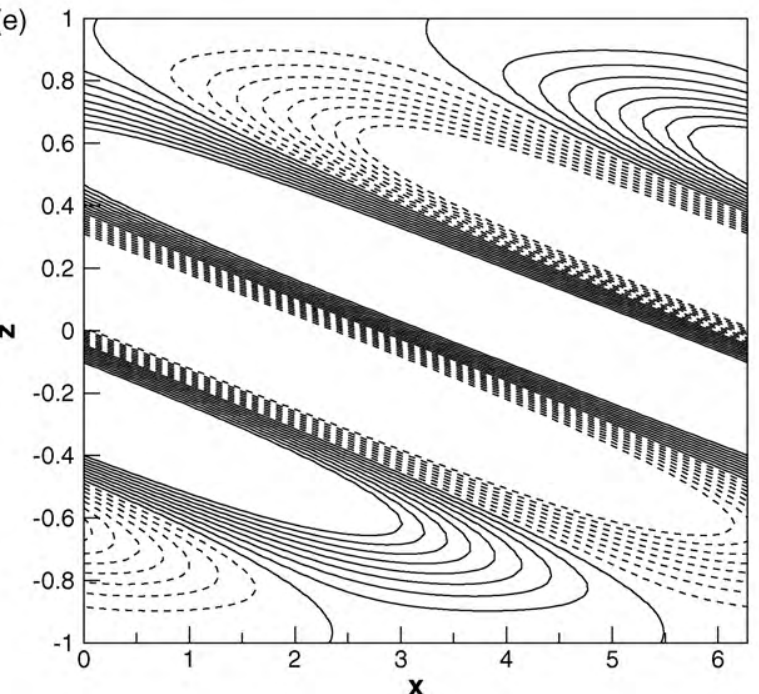

(b)

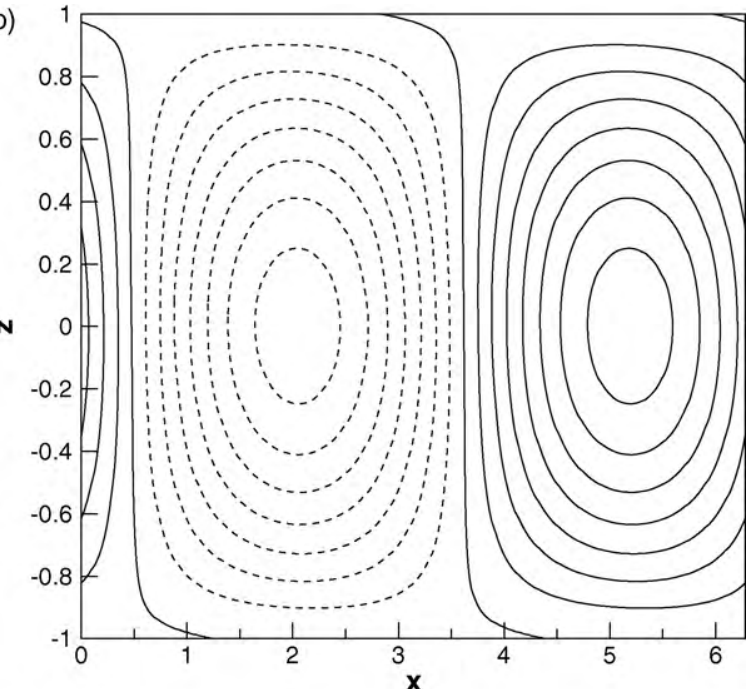

(d)

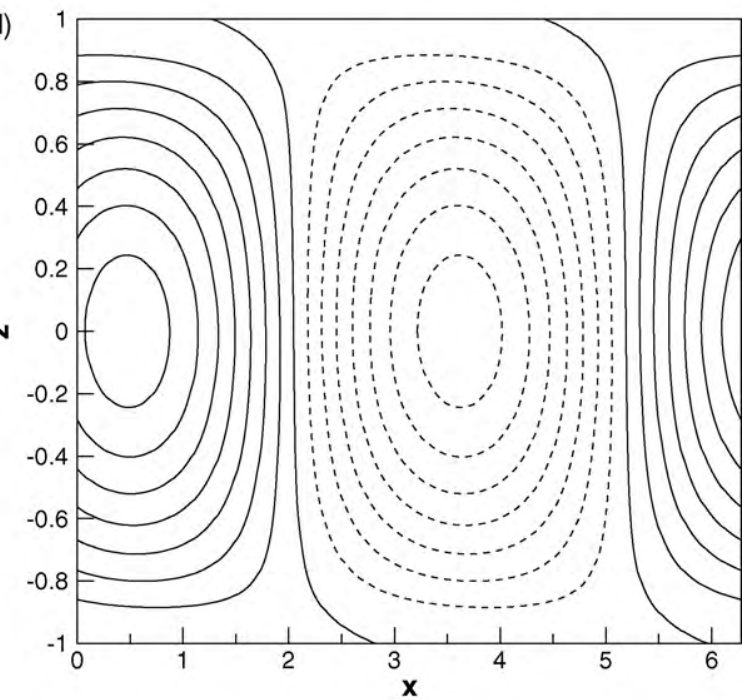

(f)

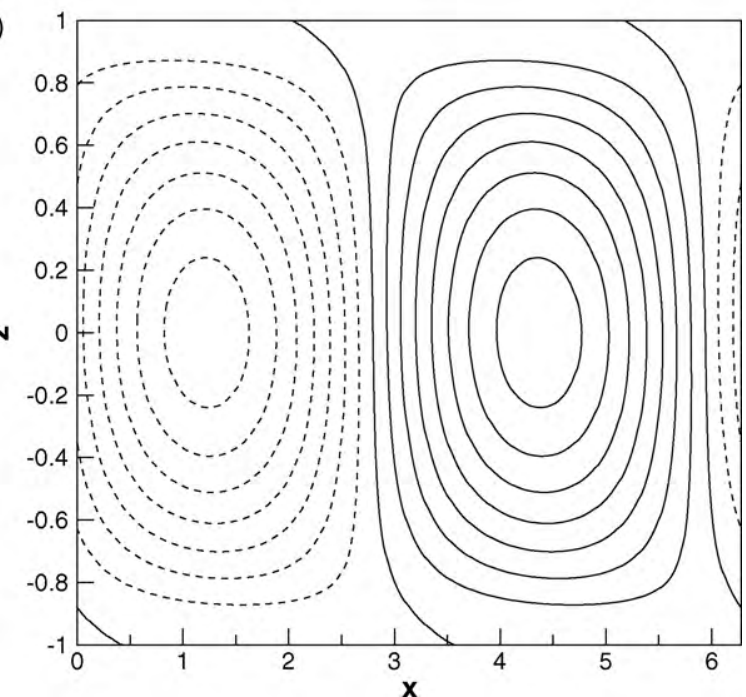

Fig. 11. Flow patterns of the streamfunction for $n=0.2,1.0$ and 2.0. (a)initial condition, (b) optimal time for $n=0.2$; (a)initial condition, (b) optimal time for $n=1.0$; (e)initial condition, (f) optimal time for $n=2.0$. 
lem, the amplitude of the streamfunction satisfies:

$\Psi(z)=\frac{i w(z)}{\alpha}$.

The streamfuction of streamwise disturbance has the form of

$\psi(x, z)=|\Psi(z)| e^{i \alpha x+i \phi(z)}$,

in which $\phi(z)$ is the argument of $\Psi(z)$. In order to know the influence of non-Newtonian viscosity on the structures of optimal disturbances, we present the amplitudes of the streamfunction and the flow patterns at initial time and optimal time for various $n$. Fixing the Reynolds number Re at 1000 and the streamwise wavenumber $\alpha$ at 1.0 , the amplitudes of streamfunction are presented in Fig. 10. As shown in Fig. 10(a), (c) and (e), at initial time both the real and imaginary parts of $\Psi(z)$ display oscillatory behavior. With the increase of $n, \Psi(z)$ becomes less oscillatory in the $z$ direction. In Fig. 10(b), (d) and (f), the oscillatory distributions of $\Psi(z)$ have been significantly smoothed. The flow patterns of the optimal disturbances of the initial time and optimal time are presented in Fig. 11. At initial time, in Fig. 11(a), (c) and (e) the disturbances are characterized by flow patterns which significantly tilt to the oppose direction of the basic flow. The flow patterns display a streak-like structure. With the increase of $n$, the widths of the streaks significantly increase. At optimal time, as shown in Fig. 11(b), (d) and (f), the structures of the flow pattern are similar.

For Couette flow, we are interested in the initial and the asymptotic long time behavior of $G(t)$. The eigenvalues of $L$ predict the asymptotic behavior of $t \rightarrow \infty$. For Couette flow there is no unstable eigenvalue, so $G(t) \rightarrow 0$ for $t \rightarrow \infty$. In Fig. 8(a), it is interesting

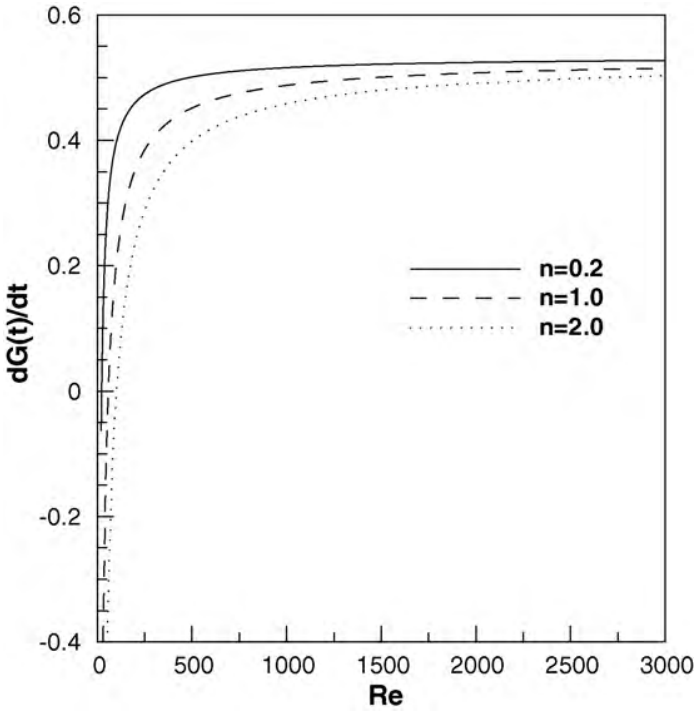

Fig. 12. $d G(t) / d t$ versus Reynolds number for various $n$.

that for small time, each curve of growth function is almost indistinguishable from other curves. In Fig. 9, similar result is found as well for various $n$ at $R e=1000$. However, in Fig. 8(b) the values of $G(t)$ for $R e=350$ is apparently lower than other curves at small time.

In order to understand the the short time behavior of $G(t)$, we examine the initial growth rate of $G(t)$ which can be given by the
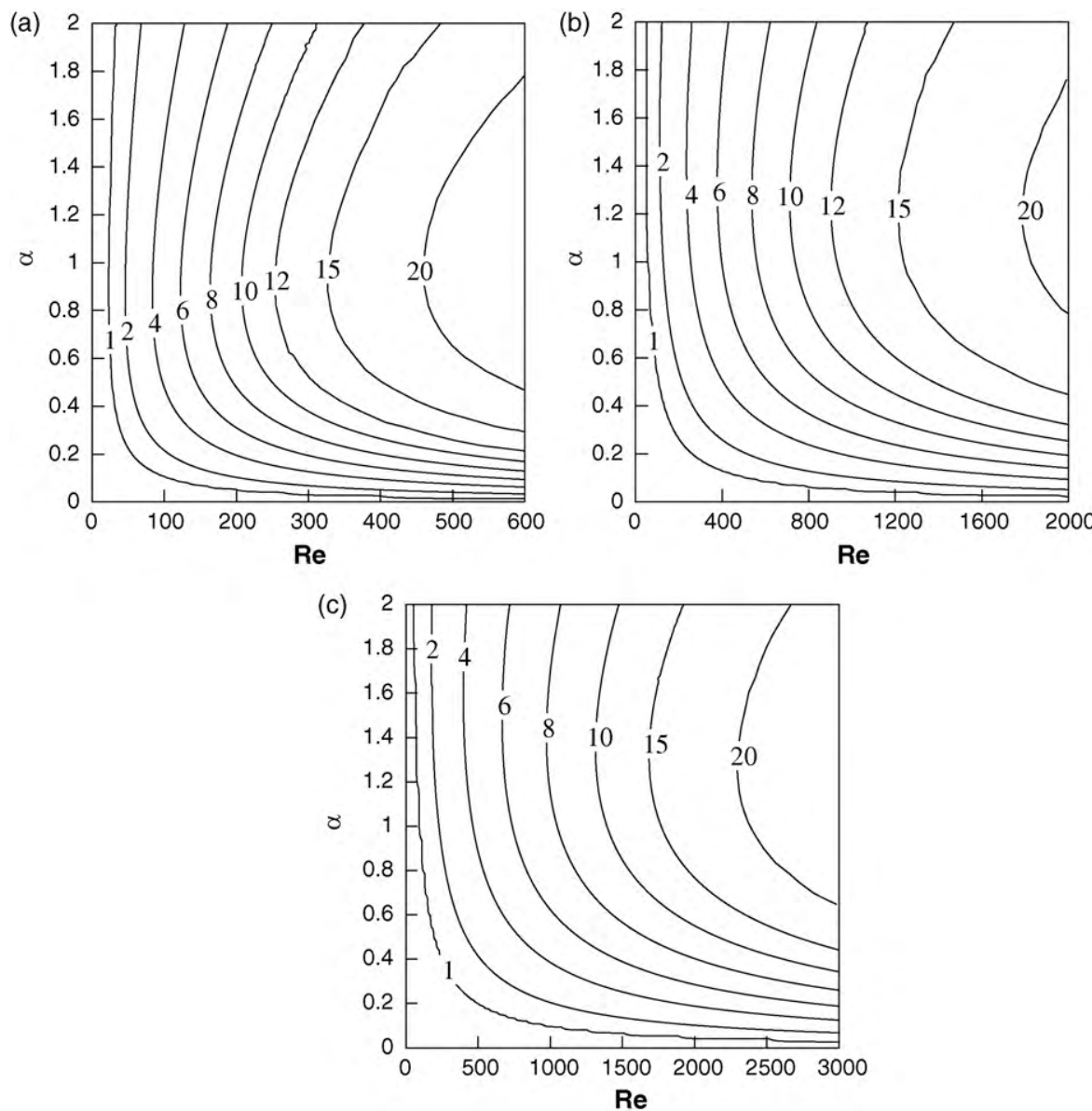

Fig. 13. Contour of $G$ for two-dimensional plane Couette flow at various $n$. (a) $n=0.2$, (b) $n=1.0$, (c) $n=2.0$. 
explicit formula:

$\left.\frac{d G(t)}{d t}\right|_{t=0}=\sup \sigma\left(-i L+i L^{*}\right)$

in which $\sigma$ denotes the set of eigenvalues. In Fig. 12 we present the curves of initial $d G / d t$ versus Reynolds number for $n=0.2,1.0$, 2.0. As shown in this figure, for each $n, d G(t) / d t$ increases dramatically at low Reynolds numbers. However, at high Reynolds numbers the $d G(t) / d t$ approximates to a constant. It is interesting that for large Reynolds number, $d G(t) / d t$ is insensitive to $n$. These results are consistent with the fact predicted in Figs. 8(a) and 9 that $G(t)$ has qualitatively the same value at small $t$.

In Figs. 8 and 9, the curves of the growth function are presented for streamwise disturbances with the wavenumber $\alpha=1.0$. In order to examine the transient behavior of the continuous operator $L$, Fig. 13(a)-(c) plots the the contours of $G(t)$ for $n=0.2,1.0,2.0$ in the $\alpha-\operatorname{Re}$ plane. The contour of $G=1$ in each figure separates the $\alpha-R e$ plane into two regions. The region of $G>1$ is linear stable but has a transient growth. As shown in Fig. 13(a) for $n=0.2$, at $R e=500$ the maximal transient growth exceeds 20. For $n=1.0$ and 2.0 in Fig. 13(b) and (c), at $R e=500$ the maximal transient growth is much lower than 20. Only when $R e$ reaches about 1800 for $n=1.0$ and 2300 for $n=2.0$, the transient growths reach 20 . This result indicates that in the region of $R e>R e_{1}$ "shear-thinning/shear-thickening" effect plays a destabilizing/stabilizing role in amplifying the transient growth.

\subsection{Influence of non-Newtonian viscosity on transient growth of spanwise disturbances}

We have studied the influences of "shear-thinning" and "shear-thickening" effects on the transient growth of streamwise disturbances. For the Couette and Poiseuille flows of Newtonian fluids, the spanwise disturbances have the optimal growth. For the present problem, we only give some results of the spanwise disturbance. The deduction of the controlling equations of spanwise disturbances can be referred in the works by Chikkad et al. [14] and by Nouar et al. [15]. In order to know the influence of "shear-thinning" and "shear-thickening" effects on the transient growth of spanwise disturbances, we present in Fig. 14 the curves of energy growth function $G(t)$ with various $n$ at $R e=1000$. As shown in this figure, with the decrease of $n$ both $G_{\max }$ and $t_{\max }$ significantly increase. This result indicates that for spanwise disturbances and streamwise disturbances, the influences of $n$ on the transient

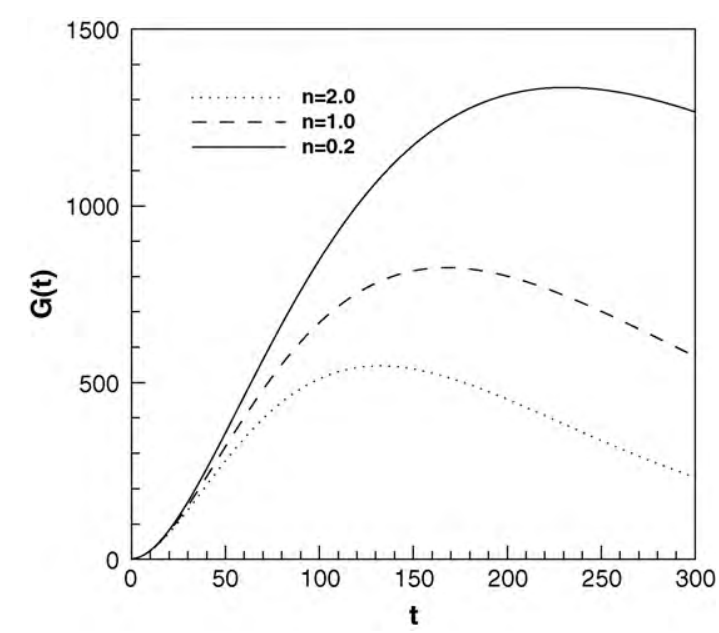

Fig. 14. Transient growth $G$ versus time for the optimal spanwise disturbance of plane Couette flow with various $n$ at $R e=1000$. The streamwise wavenumber $\alpha=0$ and the spanwise wavenumber $\beta=1.0$. growth are similar. Recently, Nouar et al. [15] have studied the influences of "shear-thinning" effect on the transient growth of spanwise disturbances in Poiseuille flow of non-Newtonian fluids using the Carreau model. The result in Ref. [15] shows that the "shear-thinning" effect slightly influences the transient growth of spanwise disturbance. If the Reynolds number is defined based on the viscosity averaged across the channel, the optimal growth of the plane Poiseuille flow significantly increases with the decrease of the index $n$. This result is qualitatively the same to that in the present paper for a plane Couette flow of a power-law fluid.

Comparing the curves of the energy growth in Fig. 14 with that in Fig. 9, it is shown that for each $n, G_{\max }$ and $t_{\max }$ of spanwise case are more larger than that of streamwise case. We look at the curves of $n=0.2$, at $t=10$ the energy growth function $G(t) \approx 20$ for streamwise case and $G(t) \approx 25$ for spanwise case. It is obvious that at the initial stage of the linear transition, the magnitudes of the energy growth of the streamwise and spanwise disturbance are comparable. This result indicates that the non-linear region may be reached before the spanwise disturbance reaches its optimal value. So, both the streamwise and the spanwise disturbances may play important roles in the non-linear transient stage.

\section{Conclusions}

The instabilities of a plane Couette flow of power-law fluid to infinitesimal two-dimensional disturbances have been investigated. We focus on the effects of non-Newtonian viscosity on the non-modal stability of streamwise disturbances. The coefficient $n>1$ and $n<1$ correspond to "shear-thinning" and "shear-thickening" liquids, respectively. The results indicate that "shear-thinning" and "shear-thickening" effects have adverse influences on the transient behaviors.

Because the evolution of infinitesimal disturbances are governed by a non-normal operator $L$, the eigenvalue analysis is inappropriate to predict the instability behavior of operator $L$. Our analysis is in the framework of non-normal stability theory rather than the traditional eigenvalue analysis. We focus on the responses to external excitations and initial conditions for both the Newtonianl and the non-Newtonian cases.

The results show that the instabilities of plane Couette flow is strongly related to the Reynolds number and the non-Newtonian coefficient $n$. Eigenvalue analysis indicates that the plane Couette flow has no unstable eigenvalue for any Reynolds number and coefficient $n$. This means the system is asymptotic stable for both the Newtonian and the non-Newtonian flows. The spectral structures of the non-Newtonian Couette flows are qualitatively similar to that of the Newtonian case, which consists of three branches. The vicinity of the intersection of the three branches becomes more sensible to perturbations with the increase of the "shear-thinning" effect.

The resolvent norm and the energy growth function $G(t)$ represent the amplitude of responses to external excitations and initial conditions. The Reynolds number and "shear-thinning/shearthickening" effect play important roles in determining the non-modal stabilities. We have examined the resonances to external excitations with a real frequency. The results show that the resonance monotonically decreases with the growth of the real frequency. We have also examined the pseudospectra for the continuous operator $L$. The plots of pseudospectra indicate that "shear-thinning" effect amplifies the responses to external excitations.

The results about the responses to initial conditions show that when the Reynolds number exceeds $R e_{1}$, the system will experience a transient growth and then an exponential decay. By fixing the wavenumber at $\alpha=1.0$, we found that the value of $R e_{1}$ significantly increases with the growth of $n$. When Re exceeds about 1000, it shows that the initial growth of $G(t)$ is insensitive to the Reynolds 
number and the coefficient $n$. In order to examine the global behavior of $G(t)$, we present the contours of $G(t)$ in the Re- $\alpha$ plane for $n=0.2,1.0,2.0$. The results show that with the decrease of $n$ the system has a larger optimal response to initial conditions.

The purpose of the present study is to provide valuable insight into the roles of non-Newtonian effect on the transient behavior of plane Couette flow. However, most of the results are limited to the instabilities to two-dimensional streamwise disturbances. We also make a brief study on the spanwise case. The influences of coefficient $n$ on the transient behaviors of the spanwise disturbance and the streamwise disturbance are similar. In our further work, we will consider the non-modal stability of the three-dimensional disturbances for non-Newtonian Couette flow.

\section{Acknowledgments}

The authors thank the financial support for this research from National Natural Science Foundation of China (50890182, 10772185) and the Knowledge Innovation Program of Chinese Academy of Sciences (KGCX-SW-409).

\section{References}

[1] R.B. Bird, W.E. Stewart, E.N. Lightfoot, Transport Phenomena, 2nd edition, John Wiley \& Sons, New York, 2002.

[2] P.J. Schmid, D.S. Henningson, M.R. Khorrami, M.R. Malik, A study of eigenvalue sensitivity for hydrodynamic stability operators, Theor. Comp. Fluid Dyn. 139 (1993) 227-240.

[3] P.J. Schmid, Nonmodal stability theory, Annu. Rev. Fluid Mech. 39 (2007) 129-162.

[4] P. Drazin, W. Reid, Hydrodynamic Stability, Cambridge University Press, 1981.

[5] L.N. Trefethen, M. Embree, Spectra and Pseudospectra: The Behavior of Nonnormal Matrices and Operators, Princeton University Press, 2005.
[6] P.J. Schmid, D.S. Henningson, Stability and Transistion in Shear Flows, Springer, New York, 2001.

[7] F. Waleffe, On a self-sustaining process in shear flows, Phys. Fluids 9 (1997) 883-900.

[8] F. Waleffe, Transition in shear flows. Nonlinear normality versus non-normal linearity, Phys. Fluids 7 (1995) 3060-3066.

[9] I. Frigaard, C. Nouar, On three-dimensional linear stability of Poiseuille flow of Binghan fluids, Phys. Fluids 15 (2003) 2843-2851.

[10] S.A. Roberts, S. Kumar, Stability of creeeping Couette flow of a power-law fluid past a deformable solid, J. Non-Newtonian Fluid Mech. 139 (2006) 93-102.

[11] N.T.M. Eldabe, M.F. El-Sabbagh, M.A.-S. El-Sayed, The stability of plane Couette flow of a power-law fluid with viscous heating, Phys. Fluids 19(2007), 0941071-9.

[12] L.B. Bergström, Transient growth of small disturbances in a Jeffrey fluid flowing through a pipe, Fluid Dyn. Res. 32 (2003) 29-44.

[13] C. Nouar, N. Kabouya, J. Dusek, M. Mamou, Modal and non-modal linear stability of the plane Bingham-Poiseuille flow, J. Fluid Mech. 577 (2007) 211-239.

[14] V. Chikkadi, A. Sameen, R. Govindarajan, Preventing transition to turbulence: a viscosity stratification does not always help, Phys. Rev. Lett. 95 (2005), 264504$1-4$.

[15] C. Nouar, A. Bottaro, J.P. Brancher, Delaying transition to turbulence in channel flow: revisiting the stability of shear-thinning fluids, J. Fluid Mech. 592 (2007) 177-194.

[16] R.B. Bird, R.C. Armstrong, O. Hassager, Dynumics of Polymeric Liquids, John Wily \& Sons, New York, 1977.

[17] N. Hoda, M.R. Jovanovic, S. Kumar, Frequency responses of streamwiseconstant perturbations in channel flows of Oldroyd-B fluids, J. Fluid Mech. 625 (2009) 411-434.

[18] H.B. Squire, On the stability for three-dimensional disturvances of viscous fluid between parallel wlalls, Proc. R. Soc. Lond. Ser. A. Fluid Mech. 142 (1933) 621-628.

[19] S.C. Reddy, D.S. Henningson, Energy growth in viscous channel flows, J. Fluid Mech. 250 (1993) 209-238.

[20] C.W. Macosko, Rheology: Principles, Measurements, and Applications, VCH, New York, 1994.

[21] L.N. Trefethen, A.E. Trefethen, S.C. Reddy, T.A. Driscoll, Hydrodynamic stability without eigenvalues, Science 261 (1993) 578-584.

[22] C. Canuto, M.Y. Hussaini, A. Quarteroni, T.A. Zang, Spectral Method in Fluid Dynamics, Springer, New York, 1986. 